ISBN: 978-602-0737-28-7

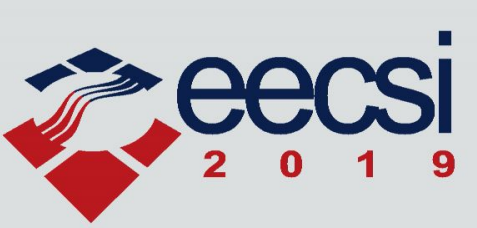

Organized by :

(3) UNERSITAS
BUDI LUHUR
Technical Co-sponsorship :

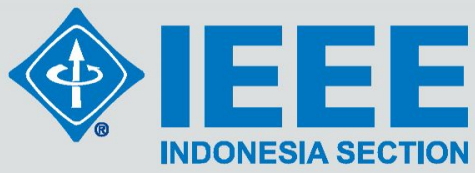

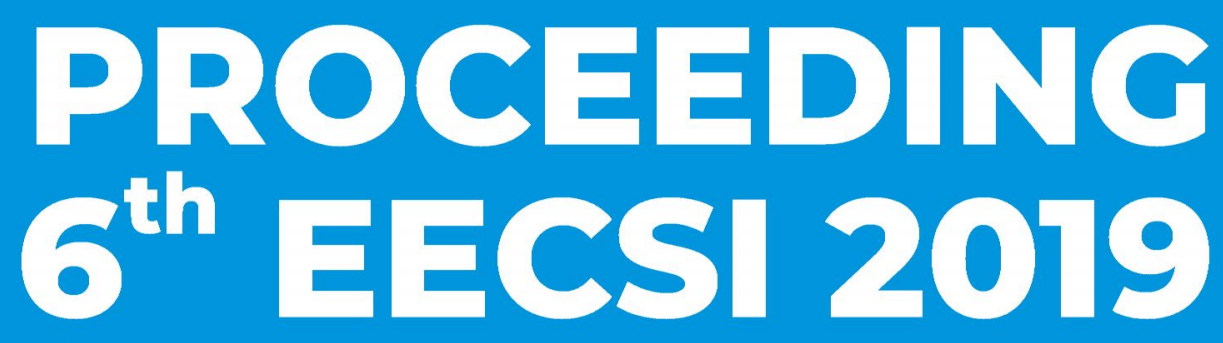

$6^{\text {th }}$ International Conference on Electrical Engineering, Computer Science and Informatics

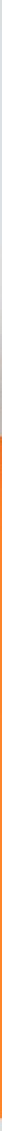

Co-organizers :

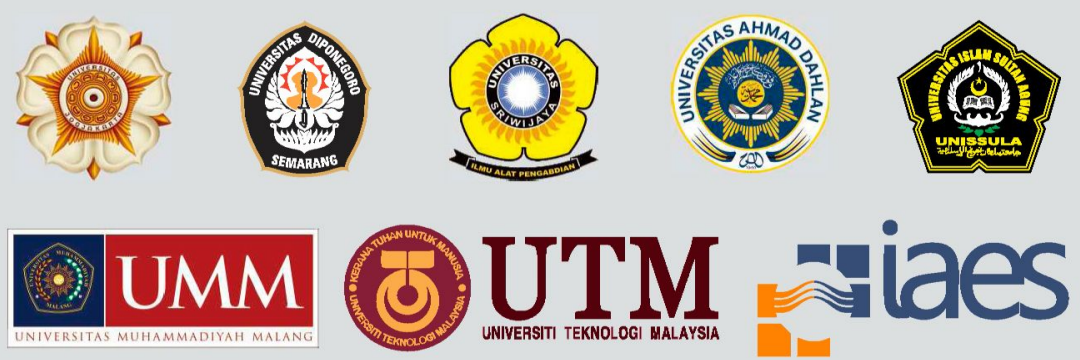




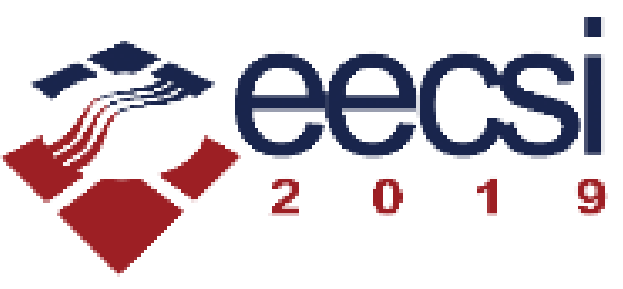

\section{PROCEEDINGS}

\section{$6^{\text {th }}$ International Conference on Electrical Engineering, Computer Science and Informatics (EECSI) 2019}

September 18 - 20, 2019, Bandung - Indonesia

\section{Editors:}

Irawan

Hendri Irawan

Munawar A Riyadi

Mochammad Facta 


\section{PROCEEDINGS}

\section{$6^{\text {th }}$ International Conference on Electrical Engineering, Computer Science and Informatics (EECSI) 2019}

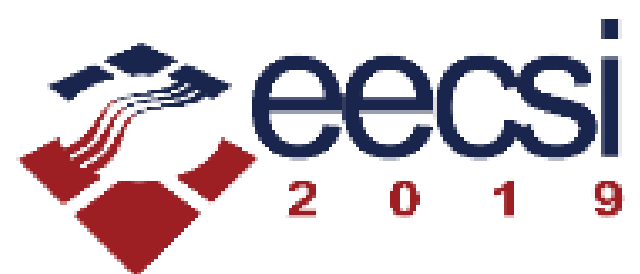

Copyright (C) 2019 Institute of Advanced Engineering and Science (IAES) All Rights Reserved

***This publication is a representation of what appears in the IEEE Digital Libraries. Some format issues inherent in the e-media version may also appear in this print version.

IEEE Catalog Number: CFP19B51-POD

ISBN : 978-602-0737-28-7 (PRINT)

ISBN : 978-602-0737-29-4 (USB)

ISBN : 978-602-0737-30-0 (DIGITAL / XPLORE FILE)

Additional Copies of This Publication Are Available From:

Curran Associates, Inc

57 Morehouse Lane

Red Hook, NY 12571 USA

Phone: (845) 758-0400

Fax: (845) 758-2633

E-mail: curran@proceedings.com

Web: www.proceedings.com 


\title{
Organizing Committee EECSI 2019
}

\author{
Advisor \\ - Pekik Argo Dahono, IEEE Indonesia Chapters Chair (EdSoc/EDS/PELS/SPS) \\ - Tumiran, Universitas Gadjah Mada, Yogyakarta, Indonesia \\ - Hermawan, Universitas Diponegoro, Semarang, Indonesia \\ - Zainudin Nawawi, Universitas Sriwijaya, Palembang, Indonesia \\ - Rahmat Budiarto, Albaha University, Baha, Saudi Arabia \\ - Sri Arttini Dwi Prasetyowati, Universitas Islam Sultan Agung, Semarang, Indonesia \\ - Kartika Firdausy, Universitas Ahmad Dahlan, Yogyakarta, Indonesia \\ - Deni Mahdiana, Universitas Budi Luhur, Jakarta, Indonesia \\ - Nazori AZ, Universitas Budi Luhur, Jakarta, Indonesia \\ - Wisnu Jatmiko, Universitas Indonesia (IEEE Indonesia Section)
}

\section{General Chair}

- Mohammad Syafrullah, Universitas Budi Luhur, Jakarta, Indonesia

\section{General Co-Chair}

- Munawar Agus Riyadi, Universitas Diponegoro, Semarang, Indonesia

\section{Finance Chairs and Treasurer}

- Wiwiek Fatmawati, Universitas Islam Sultan Agung, Semarang, Indonesia

- Widodo MS, Universitas Budi Luhur, Jakarta, Indonesia

- Martini, Universitas Budi Luhur, Jakarta, Indonesia

- Lina Handayani, Universitas Ahmad Dahlan, Yogyakarta, Indonesia

\section{Program Chairs}

- Deris Stiawan, Universitas Sriwijaya, Palembang, Indonesia

- Mochammad Facta, Universitas Diponegoro, Semarang, Indonesia

- Irawan, Universitas Budi Luhur, Jakarta, Indonesia

- Windarto, Universitas Budi Luhur, Jakarta, Indonesia

- Dewi Kusumaningsih, Universitas Budi Luhur, Jakarta, Indonesia

- Hendri Irawan, Universitas Budi Luhur, Jakarta, Indonesia

\section{Publication Chairs}

- Indra Riyanto, Universitas Budi Luhur, Jakarta, Indonesia

- Peby Wahyu Purnawan, Universitas Budi Luhur, Jakarta, Indonesia

- Tjahjanto, Universitas Budi Luhur, Jakarta, Indonesia

- Imelda, Universitas Budi Luhur, Jakarta, Indonesia

\section{Public Relations Chairs}

- Liza Dwi Ratna, Universitas Budi Luhur, Jakarta, Indonesia

\section{Local Arrangement, Exhibits \& Registration Chairs}

- Mardi Hardjianto, Universitas Budi Luhur, Jakarta, Indonesia

- Sovan Dianarto, Universitas Budi Luhur, Jakarta, Indonesia

- Dolly Virgian Shaka Yudha Sakti, Universitas Budi Luhur, Jakarta, Indonesia

- Ricky Widyananda Putra, Universitas Budi Luhur, Jakarta, Indonesia 
- Wasiran, Universitas Budi Luhur, Jakarta, Indonesia

- M. Ichsan, Universitas Budi Luhur, Jakarta, Indonesia

- Titi Hastuti, Universitas Budi Luhur, Jakarta, Indonesia

- Umaimah Wahid, Universitas Budi Luhur, Jakarta, Indonesia

- Putri Suryandari, Universitas Budi Luhur, Jakarta, Indonesia

- Titin Fatimah, Universitas Budi Luhur, Jakarta, Indonesia 


\section{Technical Program Committee}

\section{Chairs}

- Krisna Adiyarta, Universitas Budi Luhur, Jakarta, Indonesia

- Kurnianingsih, IEEE Indonesia Section

- Mochammad Facta, Universitas Diponegoro, Semarang, Indonesia

\section{Members}

- Humaira Anwer ( National University of Sciences \& Technology, Islamabad, Pakistan)

- Mahdi Baradarannia ( University of Tabriz, Iran)

- Deniz Dal ( Ataturk University, Turkey)

- Tole Sutikno ( Universitas Ahmad Dahlan, Yogyakarta, Indonesia)

- Zulfatman Has ( University of Muhammadiyah Malang, Indonesia)

- Maxime Leclerc ( Université Laval, Canada)

- Chih-Chin Liang ( National Formosa University, Taiwan)

- Evgeny Markin (, Russia)

- Agus Minarno ( Universitas Muhammadiyah Malang, Indonesia)

- Maurice Ntahobari ( Institut Teknologi Sepuluh Nopember, Indonesia)

- Naveed Sabir ( Mehran University Of Engineering \& Technology, Jamshoro, Pakistan)

- Abdulqawi Saif ( Université de Lorraine, France)

- Steffen Späthe ( Friedrich-Schiller-University Jena, Germany)

- Robert Szabolcsi ( Óbuda University, Hungary)

- Amin Torabi Jahromi ( Persian Gulf University, Iran) 


\section{Reviewers}

Nidhal Abass (Computer Science Dept., University of Kufa, Najaf, Iraq)

Elias Aboutanios (The School of Electrical Engineering and Telecommunications, University of New South Wales, Sydney, Australia)

Haitham Abu Ghazaleh (Engineering and Computer Science, Tarleton State University, Stephenville, USA)

Mohd Ashraf Ahmad (Faculty of Electrical and Electronics Engineering, Universiti Malaysia Pahang, Malaysia)

Mohd Khairul Ikhwan Ahmad (FKEE, Universiti Tun Hussein Onn Malaysia, Bt Pahat Johor, Malaysia)

Louazani Ahmed (Computer Science, Ahmed ZABANA University Center of Relizane, Algeria)

Rana Khudhair Ahmed (Computer EngineeringTechniques Department, Al-Rafidain University College, Baghdad, Iraq)

Lateeef Adesola Akinyemi (Electrical Engineering, Lagos State University, Lagos, Nigeria)

Omar Al saif (Electrical Department, Northern Technical University, Iraq)

Areej M. Abduldaim Al-Alwash (Department of Applied Sciences, University of Technology, Iraq)

Mohammad Al-Mashhadani (Dept. of Computer Techniques Engineering, Al-Maarif University College, Iraq)

Karim Al-Saedi (Computer Science Dep., Mustansiriyah University, Baghdad, Iraq)

Mohammad Al-Shabi (Department of Mechanical Engineering, University of Sharjah, United Arab Emirates)

Hamid Alasadi (COMPUTER, IRAQ- BASRA, Iraq, Iraq)

Felix Albu (Valahia University of Targoviste, Targoviste, Romania)

Shajith Ali (Electrical and Electronics Engineering, SSN College of Engineering, Chennai, Chennai, India)

Mehran Alidoost Nia (Department of Software Engineering, University of Tehran, Iran)

Farrukh Arslan (Computer Engineering, Purdue University, West Lafayette, USA)

Muhammad Sohaib Ayub (School of Science and Engineering (SSE), Lahore University of Management Sciences, Lahore, Pakistan)

Eduard Babulak (Computer Science, Liberty University, Lynchburg, USA)

Ameur Bennaoui (Department of Electronics Faculty of Electrical Engineering, University of Science and Technology (USTO), Ainrich, Algeria)

Parameshachari Bidare Divakarachari (Telecommunication Engineering, GSSSIETW, Mysuru, Visvesvaraya Technological University, Tiptur, India)

Idris bin Ismail (Electrical Engineering, Universiti Teknologi PETRONAS, Manchester, Malaysia)

Rodrigo Campos Bortoletto (Computer Science, Instituto Federal de São Paulo, Santo André, Brazil)

César Cárdenas (Mechatronics, Tecnológico de Monterrey - Campus Guadalajara, Mexico)

Su Fong Chien (ADAM, MIMOS Berhad, Kuala Lumpur, Malaysia)

Paolo Crippa (DII - Dept. of Information Engineering, Università Politecnica delle Marche, Ancona, Italy)

Bogdan Cristea (Microchip, Bucharest, Romania)

Kesavaraja D (Computer Science and Engineering, Dr Sivanthi Aditanar College of Engineering, India)

Sarada Dakua (Qatar Robotic Surgery Centre, Hamad Medical Corporation, Doha, Qatar)

Raid Daoud (Electrical Techniques, Northern Technical University/Al-Hawija Institute, Iraq)

Narottam Das (School of Engineering and Technology, CQUniversity Australia, Melbourne, Australia)

Ranjan Dash (Computer Science \& Application, College of Engineering and Technology, Bhubaneswar, India)

Mohd Daud (Mechanical Engineering, Politeknik Sultan Salahuddin Abdul Aziz Shah, Batu Pahat, Malaysia)

Tresna Dewi (Electrical Engineering, Politeknik Negeri Sriwijaya, Palembang, Indonesia)

Dan Dobrea (Faculty of Electronics and Telecommunications, Technical University "Gh. Asachi", Iasi, Romania)

Hela Elmannai (Princess Nourah University, KSA, Tunisia)

Nibras Faqera (Computer Science, Universiti Sains Malaysia, Penang, Malaysia)

Muftah Fraifer (Computer Science and Information Systems Department, IDC-CSIS-UL, Limerick, Ireland) 
Madhu Ghattamaneni (Electronics and Control Engineering, Sree Vidyanikethan Engineering College, Chandragiri Mandal, India)

Konstantinos Giannakis (Informatics, Ionian University, Edessa, Greece)

Ezra Morris Gnanamuthu (Electrical and Electronics, Universiti Tunku Abdul Rahman, Kuala Lumpur, Malaysia)

Renaldi Gondosubroto (GReS Studio, Jakarta, Indonesia)

Renliang Gu (Google Inc., Mountain View, USA)

Yuchun Guo (School of Electrical and Information Engineering, Beijing Jiaotong University, Beijing, 100044, P.R. China)

Akhil Gupta (School of Electronics and Electrical Engineering, Lovely Professional University, India)

Zaher Haddad (Computer Science, Alaqsa University, Gaza, Palestine)

Seng Hansun (IT, Universitas Multimedia Nusantara, Tangerang, Indonesia)

Suneeta Harlapur (ECE, Vemana Institute of Technology, Bangalore, India)

Rini Hasanah (Electrical Engineering Department, Brawijaya University, Malang, Indonesia)

Sherief Hashima (Engineering dept, Nuclear Research Center, EAEA, Cairo, Elsanta, Egypt)

Roberto Carlos Herrera Lara (Department of Information Technology, Division of Data Networks and Communications Systems, National Polytechnic School, Quito, Ecuador)

Raaed Ibrahim (Computer, Foundation of Technical Education, Iraq)

Donato Impedovo (Dipartimento di Informatica, Università degli Studi di Bari, Italy)

Md. Moidul Islam (Center for Energy and Environmental Chemistry (CEEC), Friedrich-Schiller-Universität Jena, Jena, Germany)

Kamarulafizam Ismail (Applied Mechanics and Design, Universiti Teknologi Malaysia, Johor Bahru, Malaysia)

Hossein Jafari (Intelligent Fusion Technology, Inc., Rockville, USA)

Ramkumar Jaganathan (Computer Science, VLB Janakiammal College of Arts and Science, Coimbatore, India)

Yumnam Jayanta (Computer Science, National Institute of Electronics and Information Technology(Kolkata), Kolkata, India)

V Jyothsna (JNTUH, India)

Sandeep Kakde (Electronics Engineering, Y C College of Engineering, India)

Yogesh Kale (Computational Science and Engineering Department, North Carolina A\&T State University, Greensboro, USA)

Noraziahtulhidayu Kamarudin (School of Computing and Creative Media, University College of Technology Sarawak, Malaysia)

Saifullah Khalid (Air Traffic Management, Civil Aviation Research Organisation, Lucknow, India)

Sunil Kumar Kopparapu (TCS Innovation Lab - Mumbai, Tata Consultancy Services, Mumbai, India)

E Hari Krishna (Dept. of ECE, Kakatiya University, Warangal, India)

Cheruku Kumar (Electronics and Communication Engineering, Amity University Rajasthan, Jaipur, India)

Parmod Kumar (Electrical and Computer Engineering, Madda Walabu University, New Delhi, India)

Otavio Lavor (Electrical Engineering, UFERSA, Brazil)

Kezhi Li (Imperial College London, London, United Kingdom (Great Britain))

Xia Li (Apple, San Diego, USA)

Xiangguo Li (College of Information Science and Engineering, Henan University of Technology, Zhengzhou, P.R. China)

Idris Lim (University of Glasgow, United Kingdom (Great Britain))

Chuan-Ming Liu (Computer Science and Information Engineering, National Taipei University of Technology, Taipei, Taiwan)

Norashikin M. Thamrin (Faculty of Electrical Engineering, UiTM, University Teknologi MARA, Shah Alam, Malaysia)

Ali Mahdoum (Centre de Développement des Technologies Avancées, Algeria)

Gerino Mappatao (ECE, De La Salle University, Manila, Philippines)

Víctor Martínez (Computing Institute, Universidade Estadual de Campinas, Campinas, Brazil)

Rajeev Mathur (Electronics, Geetanjali Instt of Tech Studies, Udaipur, Udaipur, India)

Michael McGuire (Dept. of Electrical and Computer Engineering, University of Victoria, Victoria, Canada)

Shilpa Mehta (Electronics and Communication, FACULTY, Hisar, India)

Zahéra Mekkioui (Physics, University of tlemcen, Tlemcen, Algeria)

Sumita Mishra (Amity School of Engineering \& Technology, Amity University Lucknow, India) 
Suraya Mohammad (Communication Technology Section, University Kuala Lumpur - British Malaysian Institute, Gombak, Malaysia)

Rodrigo Montufar-Chaveznava (Geophysics, Facultad de Ingeniería, Universidad Nacional Autonoma de Mexico, Mexico, Mexico)

Gen Motoyoshi (NEC Corporation, Japan)

Dina Murad (Information Systems, BINUS Online Learning, Bina Nusantara University, Indonesia)

Josip Music (Faculty of Electrical Engineering, Mechanical Engineering and Naval Architecture, University of Split, Croatia)

Marwan Nafea (Department of Electrical and Electronic Engineering, University of Nottingham Malaysia, Semenyih, Malaysia)

Tomoaki Nagaoka (National Institute of Information and Communications Technology, Japan)

Nasser Najibi (Cornell University, Ithaca, USA)

Ilesanmi Oluwafemi (Department of Electrical and Electronic Engineering, Ekiti State University, Ado Ekiti, Nigeria)

Henry Palit (Informatics, Petra Christian University, Surabaya, Indonesia)

Haijun Pan (ECE Department, New Jersey Institute of Technology, Newark, USA)

Manojkumar Parmar (Engineering Technology Strategy (RBEI/ETS), Robert Bosch Engineering and Business Solutions Private Limited, Bangalore, India)

Thaksen Parvat (Computer Engineering, Sinhgad Institute of Technology, Lonavala, Lonavala, India)

Shashikant Patil (Electronics and Telecommunication Engineering \& Mechatronics Engineering and Electrical Engineering, SVKM NMIMS Mumbai India, Shirpur, India)

Tripura Pidikiti (Electrical and Electronics Engineering, R V R and J C College of Engineering, Guntur, India)

Rajesh Pindoriya (School of Computing \& Electrical Engineering (SCEE), Indian Institute of Technology Mandi, Mandi, India)

Fitri Maya Puspita (University of Sriwijaya, Indonesia)

Harikumar Rajaguru (Electronics and Communication Engineering, Bannari Amman Institute of Technology, Sathyamangalam, India)

Grienggrai Rajchakit (Mathematics, Maejo University, Thailand)

Karthikeyan Ramasamy (Electrical and Electronics Engineering, Anna University, Chennai, India)

Partha Pratim Ray (Computer Applications, Sikkim University, Gangtok, India)

Candid Reig (Electronic Engineering, University of Valencia, Burjassot, Spain)

Abdalhossein Rezai (ACECR, Isfahan, Iran)

Indra Riyanto (Electrical Engineering, Universitas Budi Luhur, Jakarta Selatan, Indonesia)

Nuno Rodrigues (Informatics and Communications Department, Instituto Politécnico de Bragança, Bragança, Portugal)

Peter Roessler (Department of Embedded Systems, University of Applied Sciences Technikum Wien, Wien, Austria)

Julio Rojas-Mora (Departament of Informatics Engineering, Universidad Católica de Temuco, Chile)

Mahmoud Rokaya (Information Systems, Taif University, Taif, Saudi Arabia)

Pawel Rozga (Institute of Electrical Power Engineering, Lodz University of Technology, Poland)

Joonas Säe (Electronical Engineering, Tampere University, Tampere, Finland)

Wael Salah (Faculty of Engineering and Technology, Palestine Technical University - Kadoorie, Tulkarm, Palestine)

Hussain Saleem (Department of Computer Science, University of Karachi, Karachi, Pakistan)

Andrews Samraj (School of Computing Science and Engineering, Mahendra Engineering College, Salem, India)

Riko Saragih (Electrical Engineering, Maranatha Christian University, Bandung, Indonesia)

Gnane Swarnadh Satapathi (Electronics and Communication Engineering, AJ Institute of Engineering and Technology, Visakhapatnam, India)

Alexander Sergienko (Dept. of Theoretical Fundamentals of Radio Engineering, St.-Petersburg Electrotechnical University, St. Petersburg, Russia)

Nadheer Shalash (Faculty of Engineering of Electrical power Techniques, Al-Mamon University College, Iraq)

Aditi Sharma (Computer Science Engineering, Quantum University, Roorkee, Uttarakhand, Kota, India)

Ajay Shukla (Information Technology, ALL India Intitute of Ayureveda(AIIA), India)

Narendra Shukla (Computer Science and Engineering, Shiv Nadar University, Gurgaon, India) 
Joni Simatupang (Electrical Engineering, President University, Cikarang Baru Jababeka, Indonesia)

Dhananjay Singh (Electronics Engineering, Hankuk University of Foreign Studies, Korea, Korea)

Rostyslav Sklyar (Independent Professional, Lviv, Ukraine)

Miguel Sovierzoski (Federal University of Technology - Parana', Curitiba, Brazil)

Nicolai Spicher (Computer Science, University of Applied Sciences and Arts Dortmund, Germany)

Ravi Subban (Computer Science, School of Engineering and Technology, Pondicherry University,

Pondicherry, Puducherry, India)

Deepak Subramanian (Group Security, AXA, France, France)

Yong Sun (Schlumberger, USA)

Sutrisno Sutrisno (Mathematics, Diponegoro University, Semarang, Indonesia)

Isha Suwalka (Electronics and Communication, CTAE, Udaipur, India)

Galandaru Swalaganata (Mathematics Education, Institut Agama Islam Negeri Tulungagung, Tulungagung, Indonesia)

Adrian Tam (Clarity Solutions Group, USA)

Ashish Tanwer (ECE, Stony Brook University, Sunnyvale, USA)

Deepti Theng (Computer Science and Engineering, G. H. Raisoni College of Engineering, Nagpur, India)

Tow Leong Tiang (School of Electrical System Engineering, Universiti Malaysia Perlis, Malaysia)

Apriana Toding (Electrical Engineering, Universitas Kristen Indonesia Paulus, Makassar, Indonesia)

Kittipong Tripetch (Electronic and Telecommunication Engineering, Rajamangala University of Technology

Suvarnabhumi, Nonthaburi, Thailand)

Madhur Upadhayay (Electrical Engineering, Shiv Nadar University, G B Nagar, India)

Prashant Upadhyaya (Electronics Department, Buddha Institute of Technology Gorakhpur, India)

Shibiao Wan (Dept. of Electronic and Information Engineering, The Hong Kong Polytechnic University, Hong Kong)

Tianhua Xu (School of Engineering, University of Warwick, Coventry, United Kingdom (Great Britain))

Apdullah Yayık (Computer Engineering, National Defense University, Ankara, Turkey)

Thaweesak Yingthawornsuk (Media Technology, King Mongkut's University of Technology Thonburi, Bangkok, Thailand)

Mohammed Younis (Computer Engineering Department, University of Baghdad, Iraq)

Pujianto Yugopuspito (Informatics, Universitas Pelita Harapan, Tangerang, Indonesia)

Mohamad Fauzi Zakaria (Mechatronics and Robotics Engineering, Universiti Tun Hussein Onn Malaysia, Batu Pahat, Malaysia)

YunWu Zhang (Southeast University, P.R. China)

Qi Zhao (Computer Science, University of California, Los Angeles, Los Angeles, USA)

Megat Zuhairi (System and Network, Universiti Kuala Lumpur, Kuala Lumpur, Malaysia) 


\section{Table of Content}

\section{6th International Conference on Electrical Engineering, Computer Science and Informatics (EECSI 2019)}

\section{INVITED SPEAKERS}

Deep Learning Approaches for Big Data Analysis (Naomie Salim (Universiti Teknologi Malaysia, Malaysia)

MAC for Internet of Things (IoT) (Shekhar Verma (Indian Institute of Information

Technology, Allahabad, India) ....

A Real-Time Visible Light Communication System on Chip Design for High Speed Wireless

Communication (Trio Adiono (STEI ITB, Indonesia))

\section{PARALLEL SESSION - ROOM 1}

Implementation of Image Segmentation Techniques to Detect MRI Glioma Tumour

(Setyawan Widyarto (Universiti Selangor, Malaysia), Siti Rafidah Kassim (Universiti

Selangor, FCVAC, Jabatan Pengkomputeran, Malaysia), Muhammad Syafrullah

(Universitas Budi Luhur, Indonesia), Widya Kumala Sari (UDL Edge, Malaysia)

Left Ventricle Heart Three Dimension Mechanical Simulation for Kinetic Energy (Mohd

Hafizulhadi Mohd Asri (Universiti Teknologi Malaysia, Malaysia \& Faculty of Industrial

Engineering Universitas Islam Sultan Agung, Indonesia), Muhammad Haikal Satria

(Universiti Teknologi Malaysia, Malaysia), Arief Marwanto (Universiti Islam Sultan

Agung (UNISSULA) Semarang, Indonesia), Mohamad Haider Abu Yazid (Universiti

Teknologi Malaysia (UTM), Malaysia)

Detection of EEG Signal Post-Stroke Using FFT and Convolutional Neural Network

(Esmeralda Contessa Djamal (Universitas Jenderal Achmad Yani, Indonesia), Widiyanti

Furi (Universitas Jenderal Achmad Yani, Indonesia), Fikri Nugraha (Universitas Jenderal

Achmad Yani, Indonesia)

Comparison of EEG Pattern Recognition of Motor Imagery for Finger Movement

Classification (Khairul Anam (University of Jember, Indonesia), Mohammad Nuh

(Institut Teknologi Sepuluh Nopember, Indonesia), Adel Al-Jumaily (Faculty of

Engineering and IT University of Technology, Sydney, Broadway NSW \& UTS - STAFF,

Australia)

Classification of Motor Imagery and Synchronization of Post-Stroke Patient EEG Signal

(Arifah Fadiyah (Universitas Jenderal Achmad Yani, Indonesia), Esmeralda Contessa

Djamal (Universitas Jenderal Achmad Yani, Indonesia)

SeizeIT: SEIZURE victims are no longer leashed (Kaushani Uthpala Kumari Ubeysingha

(SLIIT, Sri Lanka)

Early Detection Application of Bipolar Disorders Using Backpropagation Algorithm (Desti

Fitriati (Pancasila University, Indonesia)

The Improved Artificial Neural Network based on Cosine Similarity for Facial Emotion

Recognition (Kartika Candra Kirana (State University of Malang, Indonesia), Slamet

Wibawanto (State University of Malang, Indonesia), Nur Hidayah (Universitas Negeri

Malang, Indonesia), Gigih Prasetyo Cahyono (Visionet Data International, Indonesia)

Emotion and Attention of Neuromarketing Using Wavelet and Recurrent Neural Networks

(Muhammad Fauzan Ar Rasyid (Universitas Jenderal Achmad Yani, Indonesia),

Esmeralda Contessa Djamal (Universitas Jenderal Achmad Yani, Indonesia)

An SoC-Based System for Real-time Contactless Measurement of Human Vital Signs and Soft

Biometrics (Aminuddin Rizal (Universitas Multimedia Nusantara, Indonesia)

Optical Studies of Er-doped Yttrium Aluminium Garnet Phosphor Materials (Nurhakimah

Norhashim (Universiti Kuala Lumpur, Malaysian Institute of Aviation Technology,

Malaysia), Shakiba Kaveh (Cambridge University, United Kingdom (Great Britain)), 
Anthony Cheetham (Cambridge University, United Kingdom (Great Britain)), Richard

Curry (University of Manchester, United Kingdom (Great Britain))

Low-Power And High Performance Of An Optimized FinFET Based 8T SRAM Cell Design

(Nurul Ezaila Alias (Universiti Teknologi Malaysia, Malaysia), Afiq Hamzah (Universiti

Teknologi Malaysia, Malaysia), Michael Loong Peng Tan (Universiti Teknologi Malaysia,

Malaysia), Usman Ullah Sheikh (Universiti Teknologi Malaysia, Malaysia), Munawar

Riyadi (Diponegoro University, Indonesia)

Fuzzy Logic Based Incubator Temp and Humid Level Controller Prototype (Kuat Supriyadi

(Universitas Islam Sultan Agung, Indonesia), Suryani Alifah (Unissula University,

Indonesia), Arief Marwanto (Universiti Islam Sultan Agung (UNISSULA) Semarang,

Indonesia)

River Water Pollution Monitoring using Multiple Sensor System of WSNs(Case: Siak River, Indonesia) (Evizal Abdul Kadir (Universitas Islam Riau, Indonesia), Hitoshi Irie (Chiba

University, Japan), Sri Listia Rosa (Universitas Islam Riau, Indonesia)

Controlled Position Navigation of Single Degree Magnetic Levitation (Dhiraj Basnet

(Tribhuvan University, Nepal), Anusha Lamichhane (Tribhuvan University, Nepal),

Amrit Panthi (Tribhuvan University, Nepal), Bipin Lamichhane (Tribhuvan University, Nepal)

PID Controller Design for Mobile Robot Using Bat Algorithm with Mutation (BAM) (Dwi

Pebrianti (FKEE, University Malaysia Pahang, Malaysia), Luhur Bayuaji (FSKPP,

Universiti Malaysia Pahang, Malaysia), Indra Riyanto (Universitas Budi Luhur,

Indonesia), Muhammad Syafrullah (Universitas Budi Luhur, Indonesia), Nurnajmin

Qasrina Ann Ayop Azmi (FKEE, Universiti Malaysia Pahang, Malaysia)

Efficient PID Controller based Hexapod Wall Following Robot (Hendril Satrian Purnama

(Universitas Ahmad Dahlan \& Institute of Advance Engineering and Science (IAES),

Indonesia), Tole Sutikno (Universitas Ahmad Dahlan \& Universiti Teknologi Malaysia,

Indonesia), Nuryono Widodo (Universitas Ahmad Dahlan, Indonesia), Srinivasan

Alavandar (Agni College of Technology, India)

Robust PID Control Design in CPS-based Batch Distillation Column (Wirenda Sekar Ayu

(Institut Teknologi Bandung, Indonesia), Pranoto Rusmin (Bandung Institute of

Technology, Indonesia), Egi Hidayat (Bandung Institute of Technology, Indonesia)

The Kinematics and Dynamics Motion Analysis of a Spherical Robot (Tresna Dewi

(Politeknik Negeri Sriwijaya, Indonesia), Pola Risma (Sriwijaya Polytechnic, Indonesia),

Yurni Oktarina (Polytechnic Sriwijaya Palembang-Indonesia, Indonesia), Lin Prasetyani

(Politeknik Manufaktur ASTRA, Indonesia), Zarqa Mulya (Politeknik Negeri Sriwijaya,

Indonesia)

Classification of Physiological Signals for Emotion Recognition using IoT (Sadhana Tiwari

(Indian Institute of Information Technology Allahabad, India), Sonali Agarwal (Indian

Institute of Information Technology, Allahabad, India), Muhammad Syafrullah

(Universitas Budi Luhur, Indonesia), Krisna Adiyarta (Universitas Budi Luhur,

Indonesia)

Diagnosis of Smear-Negative Pulmonary Tuberculosis using Ensemble Method: A

Preliminary Research (Rusdah Rusdah (Universitas Budi Luhur, Indonesia), Mohammad

Syafrullah (Budi Luhur University, Indonesia)

PARALLEL SESSION - ROOM 2

DNSBL for Internet Content Filtering Utilizing pfSense as The Next Generation of

Opensource Firewall (Alby A Mugni (Muhammadiyah University of Sukabumi, Indonesia)

Object Distance Measurement System Using Monocular Camera on Vehicle (Fussy Mentari

Dirgantara (Bandung Institute of Technology, Indonesia)

Marine Vessel Telemetry Data Processing Using Machine Learning (Herry Susanto

(Universitas Indonesia, Indonesia), Gunawan Wibisono (University of Indonesia, Indonesia) 
Implementation of L3 Function on Virtualization Environment using Virtual Machine Approach (Marcel Yap (Krida Wacana Christian University, Indonesia)

Flatbuffers Implementation on MQTT Publish/Subscribe Communication as Data Delivery

Format (Muhammad Adna Pradana (Telkom University, Indonesia), Andrian

Rakhmatsyah (School of Computing - Telkom University, Indonesia), Aulia Arif Wardana

(Telkom University, Indonesia)

Modified Backward Chaining Android Application to Diagnose Psychoneurosis and

Psychosomatic Disorder (Wibby Aldryani Astuti Praditasari (Universitas Darma

Persada, Indonesia), Eva Novianti (Bina Nusantara University, Indonesia), Ikhwannul

Kholis (Universitas Mpu Tantular, Indonesia), Rian Andriyusadi (Universitas Darma

Persada, Indonesia)

Intelligent System for Recommending Study Level in English Language Course using CBR

Method (Utomo Budiyanto (Gadjah Mada University, Indonesia)

Testing Big Data Applications (Narinder Punn (Indian Institute of Information Technology,

Allahabad, India), Sonali Agarwal (Indian Institute of Information Technology,

Allahabad, India)

Technologies, methods, and approaches on detection system of plant pests and diseases

(Devie Rosa Anamisa (Universitas of Trunojoyo Madura, Indonesia), Muhammad Yusuf

(University of Trunojoyo, Madura, Indonesia), Wahyudi Agustiono (University of

Trunojoyo Madura, Indonesia), Mohammad Syarief (University of Trunojoyo Madura, Indonesia)

Prediction Of Students Academic Success Using Case Based Reasoning (Abdul Rahman (Budi

Luhur University, Indonesia), Rezza Anugrah Mutiarawan (Budi Luhur University, Indonesia), Agung Darmawan (Budi Luhur University, Indonesia), Yan Rianto (Lembaga Ilmu Pengetahuan Indonesia (LIPI), Indonesia), Mohammad Syafrullah (Budi Luhur University, Indonesia)

Case Based Reasoning Adaptive E-Learning System Based On Visual-Auditory-Kinesthetic Learning Styles (Utomo Budiyanto (Gadjah Mada University, Indonesia)

Securing IoT Network using Lightweight Multi-Fog (LMF) Blockchain Model (Muhammad Yanuar Ary Saputro (University of Indonesia, Indonesia), Riri Fitri Sari (University of Indonesia, Indonesia)

An Android-based Hoax Detection for Social Media (Supardi Supardi (Universitas Budi Luhur, Indonesia), Arif Bramantoro (Universitas Budi Luhur, Indonesia), Harrizki Arie Pradana (STMIK Atma Luhur, Indonesia), Ari Amir Alkodri (STMIK Atma Luhur, Indonesia), Okkita Rizan (STMIK Atma Luhur, Indonesia), Tri Sugihartono (Stmik Atma Luhur, Indonesia)

Forecasting Indonesia Composite Index Using the Optimization of Fuzzy Backpropagation Neural Network (Anwar Rifai (Universitas Budi Luhur, Indonesia)

Fractals Study and Its Application (Setyawan Widyarto (Universiti Selangor, Malaysia), Muhammad Syafrullah (Universitas Budi Luhur, Indonesia), Gilang Budaya (Universitas Gadjah Mada, Indonesia), Muhammad Widyo Sharif (Universitas Indonesia, Malaysia)

Design and Implementation of MPC for Energy Optimization of Boiler in Batch Distillation Column (Handy Harjamulya (Institut Teknologi Bandung, Indonesia), Pranoto Rusmin (Bandung Institute of Technology, Indonesia), Egi Hidayat (Bandung Institute of Technology, Indonesia), Arief Syaichu-Rohman (Institut Teknologi Bandung, Indonesia) ........205

Analysis and Development of Information Security Framework for Distributed E-

Procurement System (Sugianto Sugianto (University of Indonesia, Indonesia), Muhammad Salman (Universitas Indonesia, Indonesia), Yohan Suryanto (Universitas Indonesia, Indonesia)

Comparison of Decision Tree, Naïve Bayes and K-Nearest Neighbors for Predicting Thesis Graduation (Achmad Solichin (Universitas Budi Luhur, Indonesia)

Spatial Coordinate Trial: Converting Non-Spatial Data Dimension for DBSCAN (Eka Arriyanti

(STMIK Widya Cipta Dharma, Indonesia), Ita Arfyanti (STMIK Widya Cipta Dharma, Indonesia), Pitrasacha Adytia (STMIK Widya Cipta Dharma, Indonesia) 
Genetic Algorithm With Random Crossover and Dynamic Mutation on Bin Packing Problem (Muhammad Syafrullah (Universitas Budi Luhur, Indonesia)

\section{PARALLEL SESSION - ROOM 3}

Civil Servant's E-Government Adoption Levels: Are age and context matters? (Iman

Sudirman (Bandung Institute of Technology, Indonesia), Atya Nur Aisha (Institut

Teknologi Bandung, Indonesia), Joe Monang (Institut Teknologi Bandung, Indonesia),

Ilham Prasetyo (Institut Teknologi Bandung, Indonesia)

Keystroke-Level Model to Evaluate Chatbot Interface for Reservation System (Supriyanto

Supriyanto (Universitas Ahmad Dahlan, Indonesia), Adhi Prahara (Universitas Ahmad

Dahlan, Indonesia)

Boosting E-Service Quality through IT Service Management of Online Stores (Sandy Kosasi

(STMIK Pontianak, Indonesia), Vedyanto Vedyanto (Santu Petrus Junior High School,

Indonesia), I Dewa Ayu Eka Yuliani (STMIK Pontianak, Indonesia)

The Quality of e-Village Budgeting Service: An Empirical Research in Banyuwangi, Indonesia

(Beny Prasetyo (Jember University, Indonesia), Saiful Bukhori (Universitas Jember,

Indonesia), Dwiky Bagas Regio Perkasa (University of Jember, Indonesia)

Implementation of Role-Based Access Control on OAuth 2.0 as Authentication and

Authorization System (Zehan Triartono (Telkom University, Indonesia), Ridha Negara

(Telkom University, Indonesia), Sussi Sussi (Telkom University, Indonesia)

Design and Implementation of Web-based Church Information Systems (Case Study: HKBP

Kebon Jeruk) (Armando Ondihon Kristoper Purba (Universitas Budi Luhur, Indonesia),

Supardi Supardi (Universitas Budi Luhur, Indonesia), Ernawati Dewi (Universitas Budi

Luhur, Indonesia), Meilieta Anggriani Porrie (Universitas Budi Luhur, Indonesia),

Muhammad Syafrullah (Universitas Budi Luhur, Indonesia)

Analysis User Readiness Level Of e-Government Using STOPE Framework (Windi Retnani

(Universitas Jember, Indonesia), Beny Prasetyo (Jember University, Indonesia), Ricky

Pangestu (Jember University, Indonesia)

E-Commerce Delivery Order System Based On ISO 9126 Model In Jeddah Saudi Arabia City

(Siswanto Siswanto (University Budi Luhur Jakarta \& Ikatan Ahli Informatika

Indonesia(IAII), Indonesia), H. Riefky Sungkar (Universitas Budi Luhur, Indonesia)

Lightweight Method for Detecting Fake Authentication Attack on Wi-Fi (Muhammad Yusuf

Setiadji (Sekolah Tinggi Sandi Negara, Indonesia, Indonesia), Ramadhan Ibrahim (Badan

Siber dan Sandi Negara, Indonesia, Indonesia), Amiruddin Amiruddin (Sekolah Tinggi

Sandi Negara \& Badan Siber dan Sandi Negara, Indonesia)

Enhancing IPSec Performance in Mobile IPv6 Using Elliptic Curve Cryptography (Supriyanto

Praptodiyono (Universitas Sultan Ageng Tirtayasa \& National Advanced IPv6 Centre,

USM, Indonesia)

Applying MAC Address-Based Access Control for Securing Admin's Login Page (Bintang

Maulana Prasetya Pagar Alam (Sekolah Tinggi Sandi Negara \& Badan Siber dan Sandi

Negara, Indonesia), Rycka Septiasari (Sekolah Tinggi Sandi Negara, Indonesia),

Amiruddin Amiruddin (Sekolah Tinggi Sandi Negara \& Badan Siber dan Sandi Negara,

Indonesia)

Optimizing Design of Core-clad Width for Single Mode Fiber with Zero Dispersion Shift

(Toto Saktioto (Universitas Riau, Pekanbaru \& Universiti Teknologi Malaysia,

Indonesia), Doni Basdyo (Universitas Riau, Indonesia), Yoli Zairmi (Universitas Riau,

Indonesia), Romi Fadli Syaputra (Universitas Riau, Indonesia), Okfalisa Okfalisa

(University Islamic Suska Riau, Indonesia), Wresni Anggraini (UIN Sultan Syarif Kasim

Riau, Indonesia), Syamsudhuha Syamsudhuha (Universitas Riau, Indonesia)

Design-of-Experiment Based Systematic Tuning of Square Open Loop Resonator (Teguh

Prakoso (Diponegoro University, Indonesia), Imam Santoso (University of Diponegoro,

Indonesia), Munawar Riyadi (Diponegoro University, Indonesia)

Optimization Info Rate Using APSK Modulation Scheme for Delivery ABIS over Satellite

Communications (Hillman Akhyar Damanik (Budi Luhur University, Indonesia), Merry

Anggraeni (Budi Luhur University, Indonesia) 
Performance Analysis of SM-MISO with Q-CSIT in Wireless Sensor Network (Subuh Pramono (Universitas Sebelas Maret, Indonesia)

Client Side Channel State Information Estimation for MIMO Communication (Sambhavi

Tiwari (Indian Institute of Information Technology Allahabad, India), Abhishek Singh

(Indian Institute of Information Technology Allahabad, India), Shekhar Verma (Indian

Institute of Information Technology, Allahabad, India), Krishna Pratap Singh (Indian

Institute of Information Technology Allahabad, India), Muhammad Syafrullah

(Universitas Budi Luhur, Indonesia), Krisna Adiyarta (Universitas Budi Luhur,

Indonesia)

Comparison of PIC and SIC with Lattice Reduction cancellation schemes for V-BLAST MIMO system (Evgeny Goncharov (JSC "SDN Soft", Skolkovo Innovations Center, Russia)

Performance Analysis of Smartphone-based Mobile Wi-Fi Hotspots Operating in a

Congested Environment (Osama M. F. Abu-Sharkh (Princess Sumaya University For

Technology, Jordan)

Spatial Diversity Impact in Mobile Quantisation Mapping for Cognitive Radio Networks

(Arief Marwanto (Universiti Islam Sultan Agung (UNISSULA) Semarang, Indonesia)

Interference Management in Heterogeneous Network With Particle Swarm Optimization

(Rummi Sirait (Universitas Budi Luhur, Indonesia), Nifty Fath (Universitas Budi Luhur, Indonesia)

Line Detection Using Arranging Coordinate Point Method (Rumaisa Ramadhani (Institut

Teknologi Bandung, Indonesia), Arief Syaichu Rohman (Institut Teknologi Bandung,

Indonesia), Yulyan Wahyu Hadi (Institut Teknologi Bandung, Indonesia)

\section{PARALLEL SESSION - ROOM 4}

Hybrid Improved Differential Evolution and Spline-based Jaya for Photovoltaic MPPT

Technique (Khusnul Hidayat (University of Brawijaya \& University of Muhammadiyah

Malang, Indonesia), Rini Nur Hasanah (Brawijaya University \& Faculty of Engineering,

Indonesia), Hadi Suyono (Brawijaya University, Indonesia)

MPPT System Using Incremental Conductance for Solar Cell in Normal and Partial Shading

Conditions (Rummi Sirait (Universitas Budi Luhur, Indonesia), Pramudya Widyantoro

(Universitas Budi Luhur, Indonesia), Akhmad Musafa (Universitas Budi Luhur, Indonesia)

Maximum Power Point Tracking in PV Arrays with High Gain DC-DC Boost Converter

(Arsyad Cahya Subrata (Universitas Ahmad Dahlan, Indonesia), Tole Sutikno

(Universitas Ahmad Dahlan \& Universiti Teknologi Malaysia, Indonesia), Sanjeevikumar

Padmanaban (Alborg University, Denmark), Hendril Satrian Purnama (Universitas

Ahmad Dahlan \& Institute of Advance Engineering and Science (IAES), Indonesia)

Dual Carrier PWM Inverter-Fed Nine-Phase AC Motors (Anwar Muqorobin (Indonesian Institute of Sciences, Indonesia), Pekik Argo Dahono (Institute of Technology Bandung, Indonesia), Estiko Rijanto (Indonesian Institiute of Sciencces, Indonesia)

Performance improvement of MO surge arrester using high gradient arrester block against VFTOs (Kannadasan Raju (Sri Venkateswara College of Engineering \& Anna University, India) ...

Performance Evaluation of Superstate HMM with Median Filter For Appliance Energy Disaggregation (Erwin Nashrullah (Universitas Indonesia, Indonesia), Abdul Halim (Universitas Indonesia, Indonesia)

Determination Of Appropriate Extra High Voltage Overhead Line Insulator (Arpan Zaeni

(ITB, Indonesia), Umar Khayam (Institut Teknologi Bandung, Indonesia), Deni

Viviantoro (ITB, Indonesia)

OTEC potential studies for energy sustainability in Riau Islands (Ibnu Kahfi Bachtiar

(Universitas Maritim Raja Ali Haji, Indonesia)

Fish Eggs Calculation Models Using Morphological Operation (Syaipul Ramdhan (STMIK

BINA SARANA GLOBAL, Indonesia), Muhammad Syafrullah (Universitas Budi Luhur, Indonesia) 
A Third Order based Additional Regularization in Intrinsic Space of the Manifold (Rakesh Kumar Yadav (IIIT Allahabad, India), Abhishek Singh (Indian Institute of Information Technology Allahabad, India), Shekhar Verma (Indian Institute of Information Technology, Allahabad, India), Venkatesan S (IIIT Allahabad, India), Muhammad Syafrullah (Universitas Budi Luhur, Indonesia), Krisna Adiyarta (Universitas Budi Luhur, Indonesia)

Speaker and Speech Recognition Using Hierarchy Support Vector Machine and

Backpropagation (Asti Fath Fadlilah (Universitas Jenderal Achmad Yani, Indonesia),

Esmeralda Contessa Djamal (Universitas Jenderal Achmad Yani, Indonesia) 404

Privacy Control in Social Networks by Trust Aware Link Prediction (Syam Dhannuri (Indian Institute of Information Technology, Allahabad, India), Sanjay Kumar Sonbhadra (Indian Institute of Information Technology, Allahabad, India), Sonali Agarwal (Indian Institute of Information Technology, Allahabad, India), P. Nagabhushan (Indian Institute of Information Technology, Allahabad, India), Muhammad Syafrullah (Universitas Budi Luhur, Indonesia), Krisna Adiyarta (Universitas Budi Luhur, Indonesia) 410

The Feasibility Of Credit Using C4.5 Algorithm Based On Particle Swarm Optimization Prediction(Siswanto Siswanto (University Budi Luhur Jakarta \& Ikatan Ahli Informatika Indonesia(IAII), Indonesia), Abdussomad Abdussomad (STMIK Nusa Mandiri, Indonesia), Windu Gata (STMIK Nusa Mandiri, Indonesia), Nia Kusuma Wardhani (Faculty of Computer Science Mercu Buana University, Indonesia), Grace Gata (Faculty of Information Technology Budi Luhur University Jakarta, Indonesia), Basuki Prasetyo (Universitas Budi Luhur, Indonesia)

Smart Performance Measurement Tool in Measuring The Readiness of Lean Higher Education Institution (Okfalisa Okfalisa (University Islamic Suska Riau, Indonesia), Fitri Insani (Assistant Lectures, Indonesia), Rahmad Abdillah (Universitas Islam Negeri Sultan Syarif Kasim Riau, Indonesia), Wresni Anggraini (UIN Sultan Syarif Kasim Riau, Indonesia), Toto Saktioto (Universitas Riau, Pekanbaru \& Universiti Teknologi Malaysia, Indonesia).

Obtaining Reference's Topic Congruity in Indonesian Publications using Machine Learning Approach (Sam F Chaerul Haviana (Universitas Islam Sultan Agung, Indonesia), Imam Much Ibnu Subroto (Universitas Islam Sultan Agung, Indonesia)

Paraphrase Detection Using Manhattan's Recurrent Neural Networks and Long Short-Term Memory (Achmad Aziz (Universitas Jenderal Achmad Yani, Indonesia), Esmeralda Contessa Djamal (Universitas Jenderal Achmad Yani, Indonesia), Ridwan Ilyas (Universitas Jenderal Achmad Yani, Indonesia)

Decision Support System with Simple Additive Weighting for Recommending Best Employee (Painem Painem (Universitas Budi Luhur, Indonesia), Hari Soetanto (Universitas Budi Luhur, Indonesia)

Gesture recognition by learning local motion signatures using smartphones (Prachi Agarwal (Indian Institute of Information Technology, Allahabad, India), Sanjay Kumar Sonbhadra (Indian Institute of Information Technology, Allahabad, India), Sonali Agarwal (Indian Institute of Information Technology, Allahabad, India), P. Nagabhushan (Indian Institute of Information Technology, Allahabad, India), Muhammad Syafrullah (Universitas Budi Luhur, Indonesia), Krisna Adiyarta (Universitas Budi Luhur, Indonesia)

Sugar Production Forecasting in PTPN XI using Autoregressive Integrated Moving Average (ARIMA) (Januar Adi Putra (Universitas Jember, Indonesia), Saiful Bukhori (Universitas Jember, Indonesia), Faishal Basbeth (University of Jember, Indonesia)

Anomaly Detection and Data Recovery on Mini Batch Distillation Column based Cyber Physical System (Wedar Panji Mardyaningsih (Institut Teknologi Bandung, Indonesia), Pranoto Rusmin (Bandung Institute of Technology, Indonesia), Budi Rahardjo (Institut Teknologi Bandung, Indonesia) 


\section{Efficient PID Controller based Hexapod Wall Following Robot}

\author{
Hendril Satrian Purnama \\ Dept. of Electrical Engg., \\ Universitas Ahmad Dahlan \\ Yogyakarta, Indonesia \\ 1friyan220@gmail.com
}

\author{
Tole Sutikno \\ Dept. of Electrical Engg., \\ Universitas Ahmad Dahlan \\ Yogyakarta, Indonesia \\ tole@ee.uad.ac.id
}

\author{
Nuryono Satya Widodo \\ Dept. of Electrical Engg., \\ Universitas Ahmad Dahlan \\ Yogyakarta, Indonesia \\ nuryono.sw@ee.uad.ac.id
}

\author{
Srinivasan Alavandar \\ Dept. of Electrical Engg., \\ Sri Krishna College of Tech. \\ Tamil Nadu, India \\ seenu.phd@gmail.com
}

\begin{abstract}
This paper presents a design of wall following behaviour for hexapod robot based on PID controller. PID controller is proposed here because of its ability to control many cases of non-linear systems. In this case, we proposed a PID controller to improve the speed and stability of hexapod robot movement while following the wall. In this paper, PID controller is used to control the robot legs, by adjusting the value of swing angle during forward or backward movement to maintain the distance between the robot and the wall. The experimental result was verified by implementing the proposed control method into actual prototype of hexapod robot.
\end{abstract}

Keywords - gait, hexapod, mobile robot, PID controller, wall following

\section{INTRODUCTION}

The locomotion control in mobile robots, such as wheeled robots [1]-[3] and walking robots [4]-[6], is an important task in some industrial applications. Unlike wheeled robots, legged robots have an ability to maneuver on different terrain, especially on uneven landscapes [7]. Among the various types of legged robots, a six legs robot configuration, known as hexapod robot, shows the advantages of more static stability and balance during walking, compared to biped and quadruped robots, due to the presence of three footrests while walking.

There are two main issues in controlling the movement of a hexapod robot, that is the coordination of the legs for forward movement and legs control for proper turning motion. Some evolutionary learning approaches has been proposed to generate the gait pattern to address the first issue [5], [8]-[11]. For the second issue, most studies have focused on gait control for trajectory following based on a predefined forward gait pattern. Inverse kinematics is the most popular approach to determine the control of degree of each leg [12][14]. This paper focuses on the second issue. The hexapod robot controller is designed to complete the wall-following task. To complete the task, a proportional-integral-derivative (PID) controller is proposed to improving the speed and stability of the hexapod robot movement while following the wall.

Several studies have been proposed in solving the wall following control problems of the mobile robot [1], [3], [23], [15]-[22]. Fuzzy logic controller (FLC) has become the most popular controller which is used to control the behaviour of the mobile robot movement. Some application of FLC for controlling the wall following task on mobile robot can be found on reference [5], [18]-[20], [23]-[26]. However, the FLC approach still has some limitation in controlling the mobile robot while conducting the wall following task.

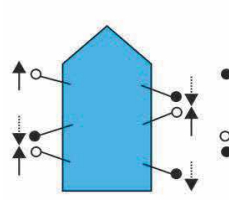

(a) o the legs is on the air

- the legs is on the ground

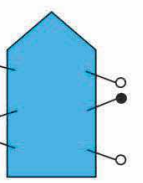

(b)

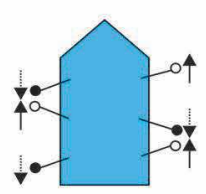

(c)

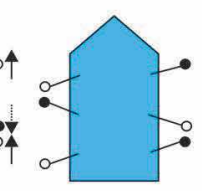

(d)
Fig. 1. The state of straightforward movement of hexapod robots

In the other case, the PID controller has been used to increase the stability and decrease the steady-state error [27]. PID controller is also the most popular techniques which are used in non-linear systems [28]. Many cases of mobile robots have used the PID controller to improve their performance [29]-[36]. In the case of wheeled based mobile robots, the PID controller is easier to implement because they are no need a complex control for actuator movement like a multileg robot.

In this paper, the design and implementation of a PID controller for the hexapod robot is proposed to improve its performance while conducting the wall following task. To verify the performance of the proposed controller, the anticipated algorithm is implemented and tested on an actual prototype of the hexapod robot and compared with the classic FLC approach.

This paper is organized as follows. Section 1 dealt with a brief introduction on hexapod robots and its various control algorithm methods. Section 2 describes the method for coordination control of hexapod robot gait. Section 3 introduces the design of PID control for wall following behaviour on hexapod robot. Section 4 presents an experimental result. Finally, section 5 presents the conclusion remarks.

\section{Hexapod Gait Coordination Control Method}

The hexapod robot has several advantage over another multi-legs robot, such as biped and quadruped robot [7], because it has more stability and balances while moving. To maintain the stability and balance of the hexapod robot, three legs should be always on the ground. The hexapod robot must have a minimum of two degrees of freedoms (DOF) per legs to complete the walking task. One DOF is used to control up or down motion, and the other DOF is used to control forward or backward motion. The optimal gait for forward movement can be controlled using a four-state finite state machine (FSM) [5]. Fig. 1. Shows the states of the six legs robot controlled using the FSM. 


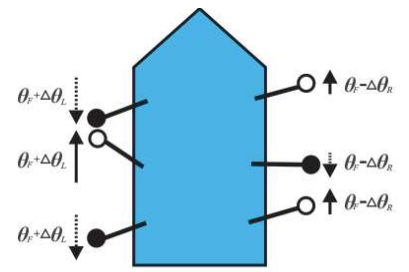

(a)

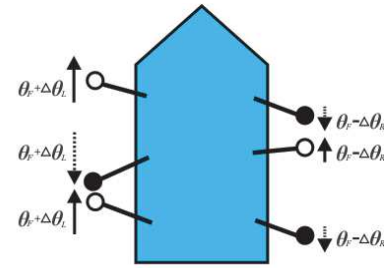

(b)
Fig. 2. The state of robot movement to make right turn

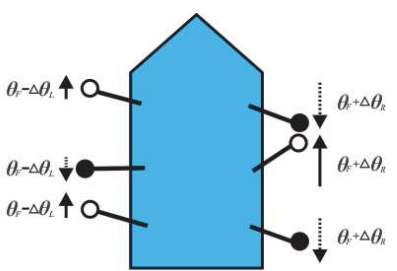

(a)

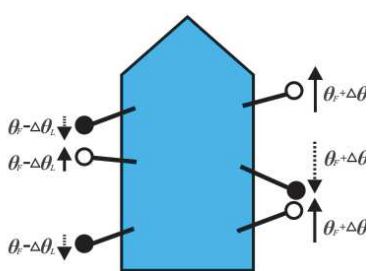

(b)
Fig. 3. The state of robot movement to make left turn

To ensure that the movement of the robot is straightforward, the swing angles $\left(\theta_{F}\right)$ of all legs are must be same in all states. Four states details of the legs control in each state are following:

- State 1 [fig. 1(a)]: Right-front, right-back, and left middle legs are the support legs. The actuator swings this leg backward, and the ground provides a reactive force to move the robot forward with a slight turn to the left side. The other three legs are on the air, with the actuator swinging that forward.

- State 2 [fig. 1(b)]: Actuators change the support legs in each state to support and up legs, respectively. That is, the up or support state of each leg in stage one is changed to the second state as seen in fig. $1(b)$.

- State 3 [fig. 1(c)]: The actuator swings three legs backward. The ground provides reactive power to move the robot forward with a slight turn to the right side, in compensation for turning left in state one; as a result, the robot moves straight forward. Then the actuator swings the other three legs forward.

- State 4 [fig. 1(d)]: The actuators change the support and up legs in state one to support and up legs, in this state. State one follows this state, and the FSM controls the robot moving straightforward.

In additional to the control of forward movement, it is also important to control the direction of the robot when carrying out the wall following tasks.

In this paper, the base algorithm that is used to coordinate the robot straightforward and turning motion is the same, the difference is only in the distance of forward or backward swing angle on the robot legs when making a turn so that the robot movement is expected to be controlled more easily. To make a right turn, the robot increases the swing angle of the left legs $\left(\Delta \theta_{L}\right)$ and decreases the swing angle of the right legs $(\Delta \theta R)$. To makes a left turn, the robot increases the swing

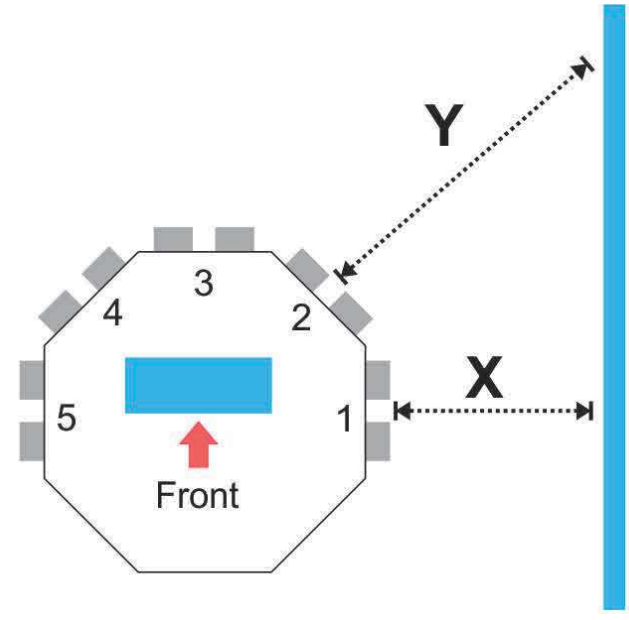

Fig. 4. Distance sensor configuration to measure the error

angle of the right legs $(\Delta \theta R)$ and decreases the swing angle of the left legs $\left(\Delta \theta_{L}\right)$. Fig. 2. shows the states of legs swing angle change to make a right turn, and Fig. 3. shows the states of legs swing angle change to make a left turn.

As the autonomous robot, the hexapod robot must have an ability to determine the proper value of $\Delta \theta_{R}$ and $\Delta \theta_{L}$ automatically. To achieve this, the PID controller is proposed in this paper to control the movement of the hexapod robot while following the wall.

\section{DESIGNING PID CONTROL FOR WALl FOLlOWING TASK OF HEXAPOD ROBOT}

This section described the design of PID controller to end the wall following task of the hexapod robot. The objective of the proposed PID controller in this paper is to improve the speed and stability of the hexapod robot movement while completing the wall following task.

Basically, the PID is a three-term controller that is used to increase stability and decrease steady-state error. The discrete function of the PID controller is given by:

$P I D=K_{p} e_{k}+K_{i} T \sum_{0}^{k} e_{k}+\frac{1}{T} K_{d}\left(e_{k}-e_{k-1}\right)$

Where $K_{P}$ is the proportional gain, $K_{I}$ is the integral gain, $K_{D}$ is the derivative gain, $\boldsymbol{T}$ is the integral time constant and $\boldsymbol{e}_{\boldsymbol{k}}$ is the accumulation of error parameter.

In the case of a wall-following task, the PID controller can be implemented by configuring the distance of sensors to accumulate the error parameter. Following the fig. 4. the error is given by:

$$
\begin{array}{r}
P V=\min (X, Y * 0.5) \\
\quad \text { Error }=S P \text { - PV; }
\end{array}
$$

Where $\mathbf{X}$ is the distance between the sensor number 1 and the wall, $\mathbf{Y}$ is the distance between the sensor number 2 and the wall, $\mathbf{S P}$ is the set-point parameter.

To get the proper values of $\Delta \boldsymbol{\theta}_{\boldsymbol{R}}$ and $\boldsymbol{\Delta} \boldsymbol{\theta} \boldsymbol{L}$, the error parameter that has been obtained from Equation (2) is substituted in the PID transfer function Equation (1), value of PID is estimated and used with the base swing angle $\left(\boldsymbol{\theta}_{\boldsymbol{F}}\right)$. Thus the value of swing angles for left and right legs are obtained as; 


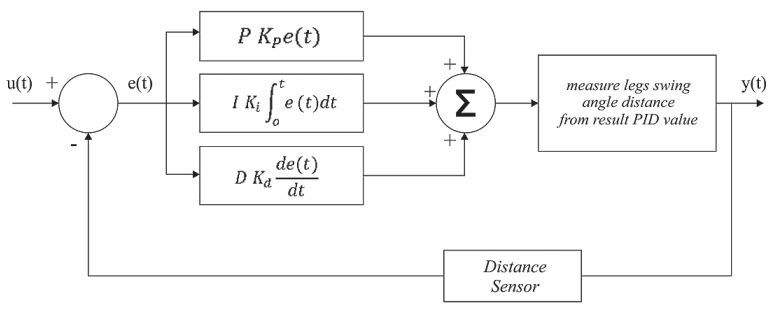

Fig. 5. Proposed PID controller diagram

$$
\begin{aligned}
& \Delta \theta_{L}=\theta F-\text { PID } \\
& \Delta \theta_{R}=\theta_{F}+\text { PID }
\end{aligned}
$$

The block diagram of the proposed PID controller in this paper is shown in fig. 5 .

Finally, the method to determine the value of $\boldsymbol{K} \boldsymbol{p}, \boldsymbol{K i}$, and $\boldsymbol{K} \boldsymbol{d}$ gain parameters of the PID controller is using the trial and error method. The tuning value is starting from $\mathbf{0}$ until 20 for each gain parameters. The best value of the PID gain parameters that we get is shown in table 1 .

TABLE I. THE BEST PID VALUE FOR THE PROPOSED CONTROLLER

\begin{tabular}{|c|c|c|c|}
\hline $\begin{array}{c}\text { Gain } \\
\text { parameters }\end{array}$ & $\boldsymbol{K} \boldsymbol{p}$ & $\boldsymbol{K i}$ & $\boldsymbol{K d}$ \\
\hline Value & 18 & 16 & 19 \\
\hline
\end{tabular}

\section{EXPERIMENTAL RESULT AND DISCUSSION}

To verify the performance of proposed PID controller, the algorithm was implemented to an actual prototype of the hexapod robot. Fig. 6. showed the real prototype of the hexapod robot that had been used. The dimension of the robot is, $23,5 \mathrm{~cm} \times 24,5 \mathrm{~cm} \times 25 \mathrm{~cm}$, for the length, width, and height, respectively. The main controller of the robot was using Arduino Due. The main of the robot actuator used digital Dynamixel AX-18A smart servo with 18 DOF. The sensor used HC-SR04 ultrasonic sensor with the configuration of the sensor shown in fig. 4.

Experimental sets were divided into two steps, first was balance and stability test and the second was speed test. For the balanced and stability test, the performance of robot was tested on the unique wall pattern. For the speed test the robot testing on a straight wall with $150 \mathrm{~cm}$ length. The proposed controller method was compared with a conventional wall following method using FLC approach [37].

TABLE II. SPEED TEST RESULT

\begin{tabular}{|c|c|c|}
\hline \multirow{2}{*}{ Attempt } & \multicolumn{2}{|c|}{ Time (s) } \\
\cline { 2 - 3 } & PID & FLC \\
\hline 1 & 5,27 & 5,59 \\
\hline 2 & 4,99 & 5,99 \\
\hline 3 & 4,94 & 5,85 \\
\hline 4 & 5,14 & 6,18 \\
\hline 5 & 5,20 & 6,26 \\
\hline Average Time (s) & $\mathbf{5 , 1 0 8}$ & $\mathbf{5 , 9 7 4}$ \\
\hline Average speed (cm/s) & $\mathbf{2 9 , 3 7}$ & $\mathbf{2 5 , 1 1}$ \\
\hline
\end{tabular}

As shown in fig. 7. the line trajectory of the hexapod robot while following the wall with the proposed PID
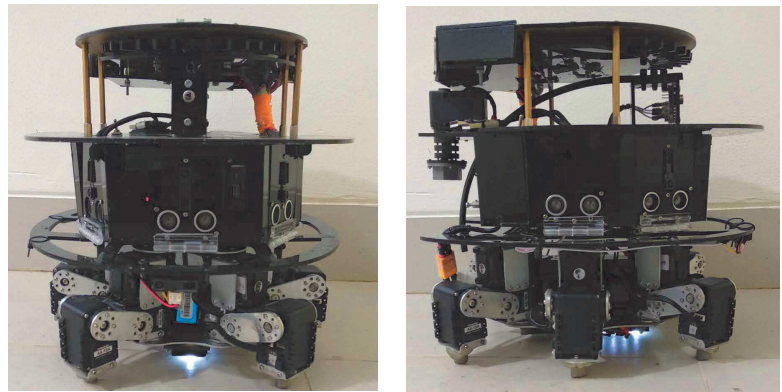

Fig. 6. Hexapod robot prototype

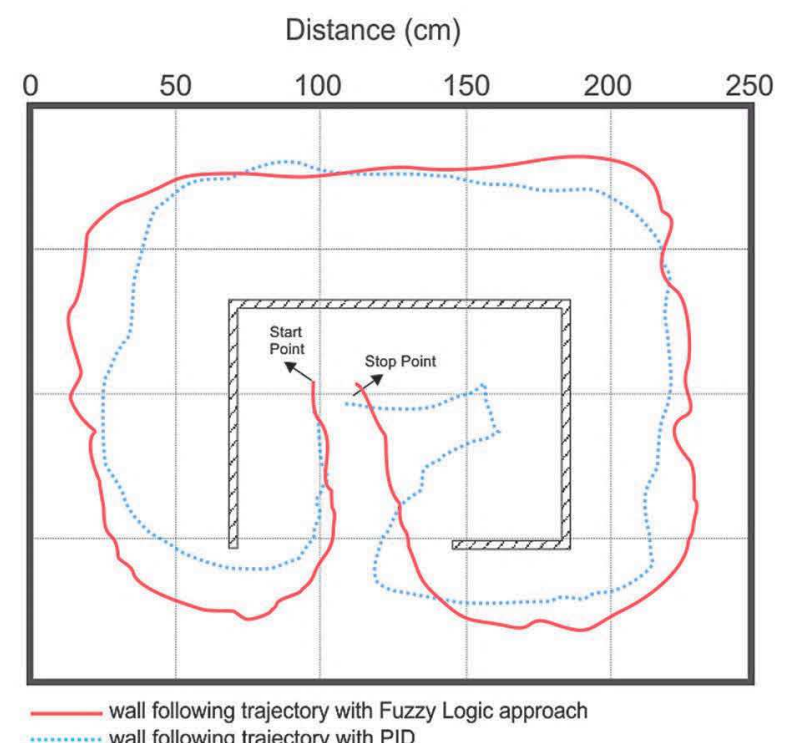

Fig. 7. Line trajectory of tested robot while following the wall
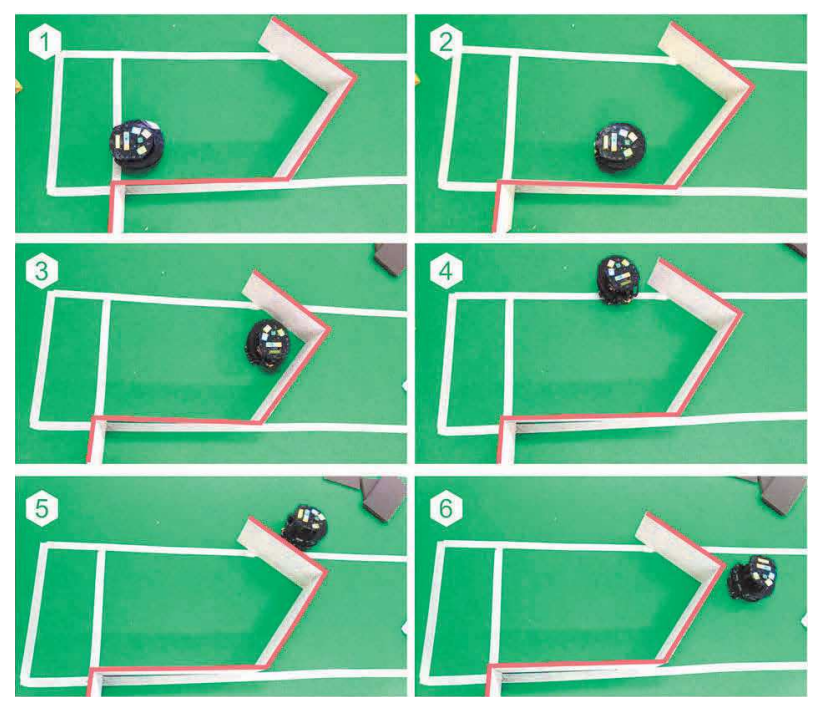

Fig. 8. Snapshot of the real hexapod robot while testing on unique environment

controller experienced more balance, straight, and close to the wall, when compared to the wall following algorithm using FLC method. That is the effect the steady-state error decrease and balance increase of a PID controller. Finally, speed test is performed on the straight wall, with $150 \mathrm{~cm}$ length and was conducted by 5 attempts to get the average speed of the proposed method. As seen in table 2. speed of the hexapod robot while using the PID controller algorithm 
was faster than using the conventional wall following algorithm.

In industrial application, the hexapod robot wallfollowing behavior can be used to move an object along the wall with an uneven floor. In other cases, hexapod robots also can be used to handle the emergency case, such as fire cases, natural disasters, etc., which requires a vehicle with the ability to move along the uneven landscapes that can't be passed by cars or another wheeled vehicle. Fig. 8. showed the snapshot of successful experimental of the hexapod robot while testing on a unique environment.

\section{CONCLUSION}

This paper had proposed a PID controller to improve the wall following performance of a hexapod robot. The PID controller is proposed to control right legs and left legs swing angles for the forward and backward movement. The proposed control method was verified by direct implementation to the actual hexapod robot. The result showed that the proposed PID controller can be used to improve the performance of the hexapod robot while conducting the wall following task.

\section{ACKNOWLEDGMENT}

This research was supported by Institute of Advance Engineering and Science (IAES) and funding by Universitas Ahmad Dahlan under Robotic Development Community research grant.

\section{REFERENCES}

[1] C. F. Juang and Y. C. Chang, "Evolutionary-group-based particleswarm-optimized fuzzy controller with application to mobile-robot navigation in unknown environments," IEEE Trans. Fuzzy Syst., 2011

[2] J. X. Xu, Z. Q. Guo, and T. H. Lee, "Design and implementation of a takagi-sugeno-type fuzzy logic controller on a two-wheeled mobile robot," IEEE Trans. Ind. Electron., 2013.

[3] B. Y. F. Cupertino, V. Giordano, D. Naso, and L. Delfine, "Implementation Using a MATLAB-Based Rapid Prototyping System," IEEE Robot. Autom. Mag., no. December, pp. 74-81, 2006.

[4] T. D. Barfoot, E. J. P. Earon, and G. M. T. D'Eleuterio, "Experiments in learning distributed control for a hexapod robot," Rob. Auton. Syst., 2006.

[5] C. F. Juang, Y. C. Chang, and C. M. Hsiao, "Evolving gaits of a hexapod robot by recurrent neural networks with symbiotic speciesbased particle swarm optimization," IEEE Trans. Ind. Electron., 2011.

[6] T. H. S. Li, Y. Te Su, S. H. Liu, J. J. Hu, and C. C. Chen, "Dynamic balance control for biped robot walking using sensor fusion, Kalman filter, and fuzzy logic," IEEE Trans. Ind. Electron., 2012.

[7] C. F. Juang, Y. H. Chen, and Y. H. Jhan, "Wall-following control of a hexapod robot using a data-driven fuzzy controller learned through differential evolution," IEEE Trans. Ind. Electron., vol. 62, no. 1, pp. 611-619, 2015

[8] R. D. Beer, "The dynamics of adaptive behavior: A research program," Rob. Auton. Syst., 1997.

[9] G. B. Parker, "Evolving gaits for hexapod robots using cyclic genetic algorithms," International Journal of General Systems. 2005.

[10] A. J. Ijspeert, "Central pattern generators for locomotion control in animals and robots: A review," Neural Networks, 2008.

[11] P. Arena, L. Fortuna, M. Frasca, and G. Sicurella, "An adaptive, self-organizing dynamical system for hierarchical control of bioinspired locomotion," IEEE Trans. Syst. Man, Cybern. Part B Cybern., 2004

[12] M. Milushev, P. Petrov, and O. Boumbarov, "Active target tracking from a six-legged robot," 2008 4th Int. IEEE Conf. Intell. Syst. IS 2008, vol. 1, pp. 456-461, 2008.

[13] M. K. Totaki, R. C. F. P. Carvalho, R. B. Letang, R. Schneiater, W. M. Moraes, and A. B. Campo, "Kinematics open loop control of hexapod robot with an embedded Digital Signal Controller (DSC)," in IEEE International Symposium on Industrial Electronics, 2010.

[14] J. Ollervides, J. Orrante-Sakanassi, V. Santibãnez, and A. Dzul, "Navigation control system of walking hexapod robot," in Proceedings - 2012 9th Electronics, Robotics and Automotive Mechanics Conference, CERMA 2012, 2012.

[15] M. J. Er and C. Deng, "Obstacle avoidance of a mobile robot using hybrid learning approach," IEEE Trans. Ind. Electron., 2005.

[16] M. Mucientes and J. Casillas, "Quick design of fuzzy controllers with good interpretability in mobile robotics," IEEE Trans. Fuzzy Syst., 2007.

[17] Y. Zhou and M. J. Er, "An evolutionary approach toward dynamic self-generated fuzzy inference systems," IEEE Trans. Syst. Man, Cybern. Part B Cybern., 2008.

[18] C. F. Juang and C. H. Hsu, "Reinforcement ant optimized fuzzy controller for mobile-robot wall-following control," IEEE Trans. Ind. Electron., vol. 56, no. 10, pp. 3931-3940, 2009.

[19] C. H. Hsu and C. F. Juang, "Evolutionary robot wall-following control using type-2 fuzzy controller with species-DE-activated continuous ACO," IEEE Trans. Fuzzy Syst., 2013.

[20] C. H. Hsu and C. F. Juang, "Multi-objective continuous-ant-colonyoptimized FC for robot wall-following control," IEEE Comput. Intell. Mag., 2013.

[21] P. van Turennout, G. Honderd, and L. J. van Schelven, "Wallfollowing control of a mobile robot," proceeding 1992 IEEE intenational Conf. Robot. Autom., no. May, pp. 280-285, 2003.

[22] R. A. Dain, "Developing Mobile Robot Wall-Following Algorithms Using Genetic Programming," Appl. Intell., 1998.

[23] R. Sanz Pedro; Ezkerra, Jose Manuel; Braunstingl, "Fuzzy Logic Wall Following of a Mobile Robot Based on the Concept of General Perception," ICAR '95, 7th Int. Conf. Adv. Robot., 1995.

[24] R. Braunstingl, J. Mujika, and J. P. Uribe, "A wall following robot with a fuzzy logic controller optimized by a genetic algorithm," in Proceedings of 1995 IEEE International Conference on Fuzzy Systems., 2002, pp. 77-82.

[25] S. F. Desouky and H. M. Schwartz, "Genetic based fuzzy logic controller for a wall-following mobile robot," in Proceedings of the American Control Conference, 2009, pp. 3555-3560.

[26] E. Tunstel and M. Jamshidi, "Embedded fuzzy logic-based wallfollowing behavior for mobile robot navigation," in Proceedings of the First International Joint Conference of The North American Fuzzy Information Processing Society Biannual Conference, 2002, pp. 329-330.

[27] Y. L. Kiam Heong Ang, Gregory Chong, "PID Control System Analysis, Design , and Technology," IEEE Trans. Control Syst. Technol., vol. 13, no. November, pp. 559-576, 2007.

[28] M. Kushwah and P. A. Patra, "Tuning PID Controller for Speed Control of DC Motor Using Soft Computing Techniques-A Review," Adv. Electron. Electr. Eng., vol. 4, no. 2, pp. 141-148, 2014.

[29] W. Cheah, H. H. Khalili, S. Watson, P. Green, and B. Lennox, "Grid-Based Motion Planning Using Advanced Motions for Hexapod Robots," in IEEE International Conference on Intelligent Robots and Systems, 2018.

[30] Z. Y. Yang, C. F. Juang, and Y. H. Jhan, "Hexapod robot wallfollowing control using a fuzzy controller," in IEEE International Conference on Control and Automation, ICCA, 2014.

[31] A. Imhof, M. Oetiker, and B. Jensen, "Wall following for autonomous robot navigation," in 2012 2nd International Conference on Applied Robotics for the Power Industry, CARPI 2012, 2012.

[32] P. van Turennout, G. Honderd, and L. J. van Schelven, "Wallfollowing control of a mobile robot," 2003.

[33] C. F. Juang and C. H. Hsu, "Reinforcement ant optimized fuzzy controller for mobile-robot wall-following control," IEEE Trans. Ind. Electron., 2009.

[34] R. Carelli and E. O. Freire, "Corridor navigation and wall-following stable control for sonar-based mobile robots," Rob. Auton. Syst., 2003.

[35] A. G. Lamperski, O. Y. Loh, B. L. Kutscher, and N. J. Cowan, "Dynamical wall following for a wheeled robot using a passive tactile sensor," in Proceedings - IEEE International Conference on Robotics and Automation, 2005.

[36] J. M. Toibero, F. Roberti, and R. Carelli, "Stable contour-following control of wheeled mobile robots," Robotica, 2009.

[37] Z. Y. Yang, C. F. Juang, and Y. H. Jhan, "Hexapod robot wallfollowing control using a fuzzy controller," IEEE Int. Conf. Control Autom. ICCA, pp. 574-578, 2014. 


\section{Authors List}

\begin{tabular}{|c|c|c|c|}
\hline Abdillah, Rahmad & 422 & Budiyanto, Utomo & $153,177,229$ \\
\hline Abdul Kadir, Evizal & 75 & Bukhori, Saiful & 253,448 \\
\hline Abdurrazaq, Ali & 286 & Cahyono, Gigih & 45 \\
\hline Abdussomad, Abdussomad & 416 & Chaerul Haviana, Sam & 428 \\
\hline Abu Yazid, Mohamad Haider & 13 & Cheetham, Anthony & 60 \\
\hline Abu-Sharkh, Osama & 325 & Curry, Richard & 60 \\
\hline Adiono, Trio & 4 & Dahono, Pekik & 363 \\
\hline \multirow[t]{2}{*}{ Adiyarta, Krisna } & $5,106,200,159$ & Damanik, Hillman & 305 \\
\hline & $315,398,442$ & Darmawan, Agung & 171 \\
\hline Adytia, Pitrasacha & 223 & Devianty, Fairuz & 40 \\
\hline Agarwal, Prachi & 442 & Dewi, Ernawati & 264 \\
\hline \multirow[t]{2}{*}{ Agarwal, Sonali } & $106,159,410$ & Dewi, Tresna & 101 \\
\hline & 442 & Dhannuri, Syam & 410 \\
\hline Agustiono, Wahyudi & 163 & Dirgantara, Fussy & 122 \\
\hline Aisha, Atya & 235 & \multirow{2}{*}{ Djamal, Esmeralda } & $18,28,49,404$, \\
\hline Alavandar, Srinivasan & 91 & & 432 \\
\hline Alias, Nurul Ezaila & 66 & Fadiyah, Arifah & 28 \\
\hline Alifah, Suryani & 71 & Fadlilah, Asti & 404 \\
\hline Al-Jumaily, Adel & 24 & Fath, Nifty & 334 \\
\hline Amir Alkodri, Ari & 189 & Firmansyah, Teguh & 286 \\
\hline Amiruddin, Amiruddin & 280,292 & Fitriati, Desti & 40 \\
\hline Anam, Khairul & 24 & Furi, Widiyanti & 18 \\
\hline Anamisa, Devie & 163 & Gata, Grace & 416 \\
\hline Andhika, Muhammad & 117 & Gata, Windu & 416 \\
\hline Andriyusadi, Rian & 147 & Goncharov, Evgeny & 320 \\
\hline Anggraeni, Merry & 305 & Halim, Abdul & 374 \\
\hline Anggraini, Wresni & 297,422 & Hamka Ibrahim, Muhammad & 311 \\
\hline Anggriani Porrie, Meilieta & 264 & Hamzah, Afiq & 66 \\
\hline Ar Rasyid, Muhammad & 49 & Harjamulya, Handy & 205 \\
\hline Arfyanti, Ita & 223 & Hasanah, Rini & 344 \\
\hline Arriyanti, Eka & 223 & Hasbullah, Iznan Husainy & 286 \\
\hline Ayop Azmi, Nurnajmin Q Ann & 85 & Herdiansah, Muhammad & 117 \\
\hline Aziz, Achmad & 432 & Hidayah, Nur & 45 \\
\hline Bachtiar, Ibnu Kahfi & 385 & Hidayat, Egi & 95,205 \\
\hline Basbeth, Faishal & 448 & Hidayat, Khusnul & 344 \\
\hline Basdyo, Doni & 297 & Hikmaturokhman, Alfin & 311 \\
\hline Basnet, Dhiraj & 80 & Ibrahim, Ramadhan & 280 \\
\hline Bayuaji, Luhur & 85 & Ilyas, Ridwan & 432 \\
\hline Bramantoro, Arif & 189 & Insani, Fitri & 422 \\
\hline Budaya, Gilang & 200 & Irie, Hitoshi & 75 \\
\hline
\end{tabular}




\begin{tabular}{|c|c|c|c|}
\hline Kassim, Siti Rafidah & 5 & Prahara, Adhi & 241 \\
\hline Kaveh, Shakiba & 60 & Prakoso, Teguh & 301 \\
\hline Khayam, Umar & 380 & Pramono, Subuh & 311 \\
\hline Kholis, Ikhwannul & 147 & Praptodiyono, Supriyanto & 286 \\
\hline Kirana, Kartika & 45 & Prasetyani, Lin & 101 \\
\hline Kosasi, Sandy & 247 & Prasetyo, Basuki & 416 \\
\hline Kumala Sari, Widya & 5 & Prasetyo, Beny & 253,270 \\
\hline Lamichhane, Anusha & 80 & Prasetyo, Ilham & 235 \\
\hline Lamichhane, Bipin & 80 & Pulasinghe, Koliya & 34 \\
\hline Lin, Yuan Hsiang & 55 & Punn, Narinder & 159 \\
\hline Listia Rosa, Sri & 75 & Purnama, Hendril & 91,358 \\
\hline Mahdiana, Deni & 195 & Putra, Januar Adi & 448 \\
\hline Mardyaningsih, Wedar & 454 & Putra, Risandi & 385 \\
\hline Marwanto, Arief & $13,71,329$ & Rahardjo, Budi & 454 \\
\hline Maspiyanti, Febri & 40 & Rahman, Abdul & 171 \\
\hline Mohd Asri, Mohd Hafizulhadi & 13 & Rahman, Abdul & 177 \\
\hline Monang, Joe & 235 & Raju, Kannadasan & 369 \\
\hline Mugni, Alby & 117 & Rakhmatsyah, Andrian & 142 \\
\hline Mulya, Zarqa & 101 & Ramadhani, Rumaisa & 338 \\
\hline Muqorobin, Anwar & 363 & Ramdhan, Syaipul & 392 \\
\hline Musafa, Akhmad & 352 & Retnani, Windi & 270 \\
\hline Mutiarawan, Rezza & 171 & Rianto, Yan & 171 \\
\hline Nagabhushan, P. & 410,442 & Ridwan, Muhammad & 117 \\
\hline Nashrullah, Erwin & 374 & Rifai, Anwar & 195 \\
\hline Negara, Ridha & 259 & Rijanto, Estiko & 363 \\
\hline Norhashim, Nurhakimah & 60 & Risma, Pola & 101 \\
\hline Novianti, Eva & 147 & Riyadi, Munawar & 66,301 \\
\hline Nugraha, Fikri & 18 & Riyanto, Indra & 85 \\
\hline Nuh, Mohammad & 24 & Rizal, Aminuddin & 55 \\
\hline Okfalisa, Okfalisa & 297,422 & Rizan, Okkita & 189 \\
\hline Oktarina, Yurni & 101 & Rusdah, Rusdah & 112 \\
\hline Ondihon K Purba, Armando & 264 & Rusmin, Pranoto & $95,205,454$ \\
\hline Osman, Azlan & 286 & S, Venkatesan & 398 \\
\hline Padmanaban, Sanjeevikumar & 358 & Saktioto, Toto & 297,422 \\
\hline Pagar Alam, Bintang Maulana & 292 & Salim, Naomie & 1 \\
\hline Painem, Painem & 438 & Salman, Muhammad & 211 \\
\hline Pandey, Ram & 80 & Santoso, Imam & 301 \\
\hline Pangestu, Ricky & 270 & Santoso, Iman & 286 \\
\hline Panthi, Amrit & 80 & Saputro, Muhammad Y A & 183 \\
\hline Pebrianti, Dwi & 85 & Saputro, Tri & 241 \\
\hline Perkasa, Dwiky & 253 & Sari, Riri & 183 \\
\hline Pradana, Harrizki & 189 & Sartana, Bruri & 229 \\
\hline Pradana, Muhammad & 142 & Satria, Haikal & 329 \\
\hline Praditasari, Wibby & 147 & Satria, Muhammad & 13 \\
\hline
\end{tabular}




\begin{tabular}{|c|c|c|c|}
\hline Sekar Ayu, Wirenda & 95 & Wardhani, Nia & 416 \\
\hline Septiasari, Rycka & 292 & Welikala, Didula & 34 \\
\hline Setiadji, Muhammad Yusuf & 280 & Wibawanto, Slamet & 45 \\
\hline Sheikh, Usman & 66 & Wibisono, Gunawan & 128 \\
\hline Singh, Abhishek & 315,398 & Widodo, Nuryono & 91 \\
\hline Singh, Krishna & 315 & Widyantoro, Pramudya & 352 \\
\hline Sirait, Rummi & 334,352 & Widyarto, Setyawan & 5,200 \\
\hline Siswanto, Siswanto & 416,274 & Widyo Sharif, Muhammad & 200 \\
\hline Soetanto, Hari & 438 & Yadav, Rakesh & 398 \\
\hline Solichin, Achmad & 217 & Yap, Marcel & 136 \\
\hline Sonbhadra, Sanjay & 410,442 & Yuliani, I Dewa Ayu Eka & 247 \\
\hline Subrata, Arsyad & 358 & Yulianti, Lenni & 122 \\
\hline Subroto, Imam Much Ibnu & 428 & Yusuf, Muhammad & 163 \\
\hline Sudirman, Iman & 235 & Zaeni, Arpan & 380 \\
\hline Sugianto, Sugianto & 211 & Zairmi, Yoli & 297 \\
\hline Sugihartono, Tri & 189 & & \\
\hline Sulaiman, Hairil & 229 & & \\
\hline Sungkar, H. Riefky & 274 & & \\
\hline Supardi, Supardi & 189,264 & & \\
\hline Supriyadi, Kuat & 71 & & \\
\hline Supriyanto, Supriyanto & 241 & & \\
\hline Suryanto, Yohan & 211 & & \\
\hline Susanto, Herry & 128 & & \\
\hline Sussi, Sussi & 259 & & \\
\hline Sutikno, Tole & 91,358 & & \\
\hline Sutrisno, Mirza & 153 & & \\
\hline Suyono, Hadi & 344 & & \\
\hline Syafrullah, Muhammad & $\begin{array}{l}5,85,106,200 \\
264,315,392\end{array}$ & & \\
\hline Syaichu Rohman, Arief & $122,205,338$ & & \\
\hline Syamsudhuha, Syamsudhuha & 297 & & \\
\hline Syaputra, Romi & 297 & & \\
\hline Syarief, Mohammad & 163 & & \\
\hline Syed Yusof, Sharifah Kamilah & 329 & & \\
\hline Tan, Michael Loong Peng & 66 & & \\
\hline Tiwari, Sadhana & 106 & & \\
\hline Tiwari, Sambhavi & 315 & & \\
\hline Triartono, Zehan & 259 & & \\
\hline Ubeysingha, Kaushani & 34 & & \\
\hline Vedyanto, Vedyanto & 247 & & \\
\hline Verma, Shekhar & 3, 315, 398 & & \\
\hline Viviantoro, Deni & 380 & & \\
\hline Wahyu Hadi, Yulyan & 338 & & \\
\hline Wardana, Aulia & 142 & & \\
\hline
\end{tabular}




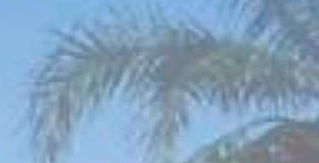

IUIDUIII)

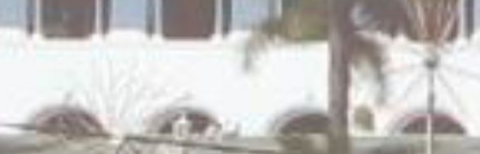

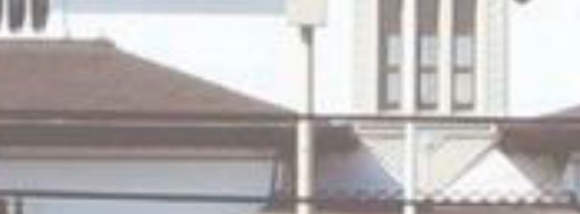

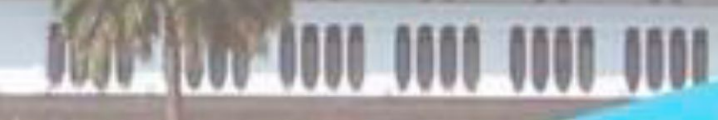

\section{$\sigma^{\text {th }}$ EECSI 2019 CONFERENCE}

September 18 - 20, 2019 ISBN 97B-602-0737-28-7 Bandung - Indonesia

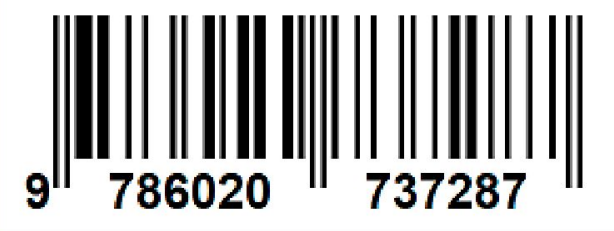

http://eecsi.org/2019 